\title{
Simulation of obliquely incident light propagation through a general twisted nematic liquid crystal cell by the Jones matrix technique
}

\author{
S. Valyukh, A. Slobodyanyuk*, V. Sorokin \\ Institute of Semiconductor Physics NASU, Kyiv, Ukraine \\ *National Taras Shevchenko University, Kyiv, Ukraine \\ E-mailsvalyukh@ksv.net.ua
}

\begin{abstract}
The simple Jones matrix technique was applied for finding intensity of obliquely incident light that passes through a plate of a uniaxial uniform medium that later was extended to general twisted nematic liquid crystal medium. Results of calculations were compared with the Berreman $4 \times 4$ matrix technique. Shown is the way of finding contrast viewing characteristics of nematic liquid-crystal displays. The obtained results are compared with experimental data.
\end{abstract}

Keywords: stratified anisotropic medium, Jones matrix, twisted nematic liquid crystal, display Paper received 15.12.99; revised manuscript received 21.01.00; accepted for publication 21.03.00.

\section{Introduction}

To understand and optimize a general twisted nematic liquid crystal display (TN-LCD), it is important to be able to mathematically simulate them. Different researchers in various ways [1-8] have calculated the transmission of polarized light through twisted nematic liquid cell (TN-LC). So far, the origin of the light guiding in the TN structures are completed, the optical properties are optimized by using empirical methods [2-4] or by complex numerical modeling, such as the Jones matrix [5-8] or the Berreman propagation matrix methods [1,9].

One of the important parameters of TN-LCD are its viewing characteristics. To obtain them, it is necessary to be able to find the transmission of obliquely incident light through TN-LCD and through retardation films (if they are). Traditionally, researchers use the Berreman method for solving such kind of problems. It suits well here, but it has an intrinsic drawback. It is enough slow. Although modern computers have a high power of calculation and the fast Berreman method was proposed [9], time of the computation is still too high for some problems. For example, there are optimization simulations that need a lot of calculations, because parameters of a system are varied. Therefore, more primitive problems, when light is incident normally, are often computed us- ing the Jones matrix method, because of the $2 \times 2$ Jones matrix technique is much more easy than the $4 \times 4$ one and, as a result, is much more fast.

In this paper we present the Jones matrix formalism for finding the electromagnetic fields propagating obliquely in layered inhomogeneous LC planar structure. The general TN-LC cell with a total thickness $L$ can be divided into $N$ thin layers. Each layer is considered as a uniaxial optical medium and has a thickness $\frac{L}{N}$. Direction of optical axis defined by molecular orientation (director) and it slightly varies from one layer to another remaining the same inside layer. The result becomes exact when $N$ is a large number. It is reasonable to assume that such system is nonmagnetic so that the magnetic permeability can be set to unity throughout all space.

In the Jones matrix method, the input polarization state and the output one are related by the following matrix equation:

$\vec{E}^{\prime}=\hat{J} \vec{E}$

where $\vec{E}=\left(\begin{array}{c}E_{x} \\ E_{y}\end{array}\right)$ is the input Jones vector, $\vec{E}^{\prime}=\left(\begin{array}{c}E_{x}^{\prime} \\ E_{y}^{\prime}\end{array}\right)$ is

the output Jones vector in a fixed coordinate system, and 
$\hat{J}_{\text {is a }} 2 \times 2$ Jones matrix. Since each of the layers of the liquid crystal can be treated as a uniform uniaxial crystal plate, the matrix $\hat{J}$ is obtained by multiplying all the Jones matrices for the plates in the sequence. First of all, we should obtain the Jones matrix of the uniform anisotropic plate. In fact, this result can be interpreted as the matrix of a retardation film. In opposite to the partial case of normal incidence, the Jones matrix for oblique incidence has more complicated expression, because the extraordinary refraction index depends on orientations of the optical axis and the direction of the light propagation. Besides, the traditional Jones matrix technique does not consider Fresnel's reflections that cannot be ignored for oblique incidence. We take into account them and find the Jones matrix of the general TN-LC cell. In order to exam the obtained results, they are compared with the Berreman method and with experimental data.

The proposed in this paper method of calculation is extremely useful for the finding of viewing characteristics of the conventional TN-LCDs, various STN, OMI, and LTN-LCDs, better understanding their electro-optical behavior and optimize the performance. In addition, it is also useful for the experimental measurement of the cell gap and both twist and tilt angles of the general TN-LCD.

\section{Uniform uniaxial crystal plate}

As a first step of our following calculations, we find the Jones matrix of a homogeneous and uniaxial crystal plate for obliquely incident light. Let $\alpha$ be the angle at which a monochromatic plane wave falls on a planar surface of a uniaxial crystal (Fig. 1). Two linearly polarized waves travel inside the crystal with different phase velocities and, in general case, in different directions. One of them is ordinary ('o') and has the refracted angle $\beta_{0}$. The other one is extraordinary ('e') and has the refracted angle $\beta_{e}$. We chose two coordinates systems. One of them $(x y z)$ is fixed. The $z$-axis is defined as the inward normal to the reflecting surface, which lies in the $x-y$ plane. The $x$ axis lies in the plane of incidence, the $y$ axis is normal to it. This coordinate system is needed for describe crystal orientation. The other one $\left(x^{\prime} y^{\prime} z^{\prime}\right)$ is not fixed, and its $z^{\prime}$ axis is parallel to the wave vector, the $y^{\prime}$ axis is the same as the $y$. This coordinate system is needed for describe of the Jones vector and matrix. Let $d$ be the thickness of a crystal with the principal refractive indexes $n_{e}$ and $n_{o}$. A unit vector $\vec{C}$ defines the orientation of its optical axis, and the wavelength of incident light is $\lambda$. After passing through anisotropic plate and analyzer two waves interfere. As far as the difference between their phase retardations is [10] $\frac{2 \pi d}{\lambda}\left(n_{o} \cos \beta_{o}-n_{e}{ }^{*} \cos \beta_{e}\right)$, we may write a relative retardation for the «o» wave as $\frac{2 \pi d n_{o} \cos \beta_{o}}{\lambda}$ and for the «e» wave as $\frac{2 \pi d n_{e}{ }^{*} \cos \beta_{e}}{\lambda}$.

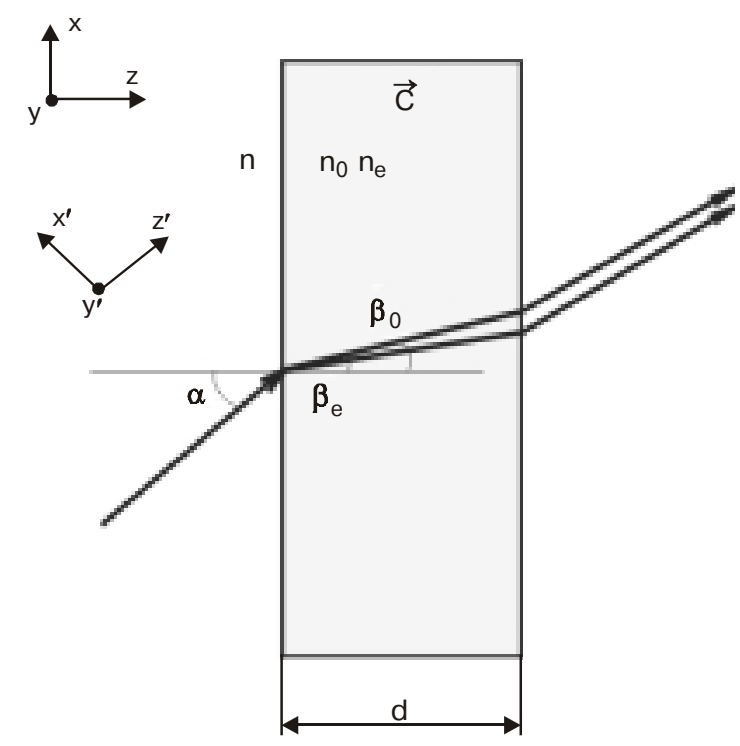

Fig. 1. Uniaxial crystal plate.

Here, $n_{e}{ }^{*}$ is the refractive index for the «e» wave in the given direction. Using the results obtained by Fedorov [11] or by Lekner [12] we may express:

$n_{e}^{*}=\sqrt{n^{2} \sin ^{2} \alpha+q_{e}^{2}}$

$q_{e}=\bar{q}-n C_{x} C_{z} \sin \alpha \Delta \varepsilon / \varepsilon_{z}$,

where $C_{i}$ are projections of vector $\vec{C}$, $\Delta \varepsilon=\varepsilon_{e}-\varepsilon_{0} \equiv n_{e}^{2}-n_{0}^{2}, \varepsilon_{z}=n_{o}{ }^{2}+C_{z}^{2} \Delta \varepsilon, n$ is the refractive index of isotropic medium, $\bar{q}^{2}=n_{o}{ }^{2}\left[n_{e}{ }^{2} \varepsilon_{z}-n^{2} \sin ^{2} \alpha\left(n_{e}{ }^{2}-C_{y}{ }^{2} \Delta \varepsilon\right)\right] / \varepsilon_{z}$.

In this way, according to $[4,5]$ the Jones matrix can be derived:

$\hat{J}=\hat{R}(\psi)\left(\begin{array}{cc}\exp \left(\frac{i 2 \pi d n_{e}^{*} \cos \beta_{e}}{\lambda}\right) & 0 \\ 0 & \exp \left(\frac{i 2 \pi d n_{o} \cos \beta_{o}}{\lambda}\right)\end{array}\right) \hat{R}^{-1}(\psi)$,

where $\hat{R}(\psi)$ is the coordinate rotation matrix, $\psi$ is the angle between plane $x^{\prime} z^{\prime}$ and optical plane (it is a plane that is formed by both the wave vector and $\vec{C}$ ). The polarization of the «o» wave is perpendicular to this plane). The angles $\beta_{o}, \beta_{e}$ are expressed through $\alpha$ by Snell's law

$\sin \beta_{e}=\frac{\sin \alpha}{n_{e}{ }^{*}} n, \quad \sin \beta_{o}=\frac{\sin \alpha}{n_{o}} n$. 


\section{S. Valyukh et al.: Simulation of obliquely incident light propagation through ...}

Finally, as far as the plane of incidence is the $x^{\prime}-z^{\prime}$ plane then $\sin \psi$ is equal to the value of $\vec{C}$ projection on $y$-axis:

$\sin \psi=C_{y}$

After substitution of Eq.(5) into Eq.(3) and few steps of matrix multiplication, we have:

$$
\begin{aligned}
\hat{J}= & A\left(\begin{array}{cc}
B\left(1-C_{y}{ }^{2}\right)+1 & B C_{y} \sqrt{1-C_{y}^{2}} \\
B C_{y} \sqrt{1-C_{y}^{2}} & B C_{y}{ }^{2}+1
\end{array}\right), \\
A= & \exp \left(i \frac{2 \pi d \sqrt{n_{o}^{2}-n^{2} \sin ^{2} \alpha} o_{o}}{\lambda}\right) \\
B= & \exp \left(i \frac { 2 \pi d } { \lambda } \left(\sqrt{n^{*} e^{2}-n^{2} \sin ^{2} \alpha}\right.\right. \\
& \left.\left.-\sqrt{n_{o}{ }^{2}-n^{2} \sin ^{2} \alpha}\right)\right)-1
\end{aligned}
$$

Now, we can find the expression for the output Jones vector $\vec{E}_{\text {out }}$ :

$$
\vec{E}_{\text {out }}=\hat{J}_{A} \hat{J} \vec{E}_{\text {in }},
$$

Where $J_{A}$ is the Jones matrix of the analyzer, $\vec{E}_{i n}$ is electrical input vector (that passed through polarize). And intensity $I_{\text {out }}$ :

$$
I_{\text {out }}=\vec{E}_{\text {out }} \vec{E}_{\text {out }}^{\times},
$$

where $\vec{E}_{\text {out }}^{\times}$is a complex conjugate vector to $\vec{E}_{\text {out }}$.

Here we should note that we have not taken into account the reflection of the wave at the interfaces of an isotropic medium and uniaxial crystal. This simplification makes all above expressions unpractical, because, as the angle of light incidence increase, the intensity of reflected wave is also increase and almost all light is reflected at large angles. So, in opposite to the case of normal incidence $[4,5]$, we cannot neglected Fresnel's reflections. For this reason, we shall use the transmission coefficients. Let $t_{o}$ and $t^{\prime}{ }_{o}$ be the coefficients for the ordinary wave of first and second boundaries between isotropic and anisotropic media, $t_{e}$ and $t^{\prime}{ }_{e}$ be the coefficients for the extraordinary wave, respectively. They are functions of the incidence angle, crystal orientation and refractive indexes. For the sake of brevity, we do not give their full expressions. Reader can easy find them in the [8,9]. Substituting these coefficients into Eq.(3) we obtain: and then

$$
\begin{aligned}
& \hat{J}=A\left(\begin{array}{cc}
B\left(1-C_{y}{ }^{2}\right)+1 & B C_{y} \sqrt{1-C_{y}^{2}} \\
B C_{y} \sqrt{1-C_{y}^{2}} & B C_{y}^{2}+1
\end{array}\right), \\
& A=t_{o} t_{o}^{\prime} \exp \left(i \frac{2 \pi d \sqrt{n_{o}^{2}-n^{2} \sin ^{2} \alpha}}{\lambda}\right), \\
& B=\frac{t_{e} t^{\prime}}{t_{o} t_{o}^{\prime}} \exp \left(i \frac { 2 \pi d } { \lambda } \left(\sqrt{n^{*} e^{2}-n^{2} \sin ^{2} \alpha}-\right.\right. \\
& \left.\left.\sqrt{n_{o}^{2}-n^{2} \sin ^{2} \alpha}\right)\right)-1
\end{aligned}
$$

In order to examine, our calculations are compared with the much more exact Berreman method. Fig. 2 shows the intensity of transmitted light through a plate of uniaxial medium $\left(n_{o}=1,5, n_{e}=1,65, d=30 \mu \mathrm{m}\right.$, $\left.C_{x}=1, C_{y}=C_{z}=0\right)$ versus the incident angle. The solid curve is the result of described above calculations, the dash curve is obtained by the Berreman method. On the whole, results obtained by both methods are in a good agreement. The unimportant difference between them is the outcome of multi-reflections that the interfaces give.

In order to obtain the exact result we should to consider them. The explicit theory of multi-interference was developed for isotropic plate [7]. Following the same procedures as these in [7], we shall find the transmission coefficients for the ordinary $\left(T_{o}\right)$ and the extraordinary $\left(T_{e}\right)$ waves:

$$
\begin{aligned}
& T_{o}=\frac{t_{o} t_{o}^{\prime}}{1-\left(1-t_{o} t_{o}^{\prime}\right) e^{-i \varphi_{o}}}, \\
& T_{e}=\frac{t_{e} t^{\prime}{ }_{e}}{1-\left(1-t_{e} t_{e}^{\prime}\right) e^{-i \varphi_{e}}} .
\end{aligned}
$$

where $\varphi_{o}, \varphi_{e}$ are the phase differences between two adjacent interfered beams for «o» and «e» waves respectively. Denoting the refraction index for the extraordinary wave that propagate toward positive values of $z^{\prime}$ axis by $n^{*} e$ and the refraction index for inverse direction by $n_{e}{ }^{*}$ (it is found the same way as $n^{*} e$ using Eq.2), we may write:

$$
\begin{aligned}
& \varphi_{o}=\frac{4 \pi d \sqrt{n_{o}{ }^{2}-n^{2} \sin ^{2} \alpha}}{\lambda}, \\
& \varphi_{e}=\frac{2 \pi d\left(\sqrt{n^{*} e^{2}-n^{2} \sin ^{2} \alpha}+\sqrt{n^{* *} e^{2}-n^{2} \sin ^{2} \alpha}\right)}{\lambda}
\end{aligned}
$$


Next, substitution Eq.11 into Eq.3 yields:

$$
\hat{J}=\hat{R}(\psi)\left(\begin{array}{cc}
T_{e} \exp \left(\frac{i 2 \pi d n_{e}{ }^{*} \cos \beta_{e}}{\lambda}\right) & 0 \\
0 & T_{o} \exp \left(\frac{i 2 \pi d n_{o} \cos \beta_{o}}{\lambda}\right)
\end{array}\right) \hat{R}^{-1}(\psi)
$$

and then

$$
\begin{aligned}
& \hat{J}=A\left(\begin{array}{cc}
B\left(1-C_{y}{ }^{2}\right)+1 & B C_{y} \sqrt{1-C_{y}^{2}} \\
B C_{y} \sqrt{1-C_{y}^{2}} & B C_{y}{ }^{2}+1
\end{array}\right) \\
& B=\frac{T_{e}}{T_{o}} \exp \left(i \frac { 2 \pi d } { \lambda } \left(\sqrt{n^{*} e^{2}-n^{2} \sin ^{2} \alpha}-\right.\right. \\
& \left.\left.-\sqrt{n_{o}{ }^{2}-n^{2} \sin ^{2} \alpha}\right)\right)-1 \\
& A=T_{o} \exp \left(i \frac{2 \pi d \sqrt{n_{o}^{2}-n^{2} \sin ^{2} \alpha}}{\lambda}\right) .
\end{aligned}
$$

We have considered the oblique incident wave propagation in the uniaxial plate and obtained the Jones matrix for this case. Substituting Eq.(14) into Eqs (7) and (8), we obtain the same result that the Berreman method yields (Fig. 2, the dash curve).

\section{Polarize}

Since a construction of TN-LC displays includes a polarize, we should find its Jones matrix. Let $\vec{k}$ be the normal vector of incident light, $\vec{c}$ corresponds to the direction in the fixed coordinate system along which the polarized electrical field is not transmitted by polarize. As far as the vectors $\vec{k}$ and $\vec{c}$ are not perpendicular in a general case, we must find the vector $\vec{p}$ that corresponds to the direction absorption in the $x^{\prime} y{ }^{\prime} z$ ' coordinate system and perpendicular to $\vec{k}$. We have:

$$
\vec{p}=\frac{[\vec{k} \times[\vec{c} \times \vec{k}]]}{|[\vec{k} \times[\vec{c} \times \vec{k}]]|}
$$

Assume that all vectors are unit. The vector $\vec{k}$ in this coordinate system has coordinates: $(0,0,1)$, the vector $\vec{c}-\left(c^{*} x, c^{*} y, c^{*} z\right)$. After vector cross multiplication we obtain the coordinates of

$$
\vec{p}=\frac{1}{\sqrt{c^{*} x^{2}+c^{*} y^{2}}}\left(\begin{array}{c}
c^{*} x \\
c^{*} y \\
0
\end{array}\right)
$$

The polarize orientation is described in the fixed coordinate system. Let $\alpha$ be the incident angle and, as result, the angle between axes $z$ and $z^{\prime}$ (Fig. 1), $\vec{c}$ is $\left(c_{x}, c_{y}, 0\right)$ in the $x y z$. Coordinates $\left(c^{*} x, c^{*} y, c^{*} z\right)$ and $\left(c_{x}, c_{y}, 0\right)$ are connected ones with others by transform matrix. After few steps of matrix manipulation we have:

$$
\vec{p}=\frac{1}{\sqrt{c_{x}{ }^{2} \cos ^{2} \alpha+c_{y}^{2}}}\left(\begin{array}{c}
c_{x} \cos \alpha \\
c_{y} \\
0
\end{array}\right) .
$$

The Jones matrix is described in the unfixed coordinate system, and we use it below. If electrical vector of incident light has coordinates $\left(E_{x}, E_{y}, 0\right)$, then the passed through polarize output one is parallel to $\vec{p}$ and may be expressed:

$\vec{E}_{\text {out }}=\frac{1}{\sqrt{c_{x}^{2} \cos ^{2} \alpha+c_{y}^{2}}}\left(\begin{array}{c}c_{x} \cos \alpha \\ c_{y} \\ 0\end{array}\right)$ (18)

Knowing the input and output electrical vectors, we may now find the Jones matrix that corresponds to Eq.(1):

$\hat{J}_{A}=\frac{1}{c_{x}{ }^{2} \cos ^{2} \alpha+c_{y}{ }^{2}}\left[\begin{array}{cc}c_{x}{ }^{2} \cos ^{2} \alpha & c_{y} c_{x} \cos \alpha \\ c_{y} c_{x} \cos \alpha & c_{y}{ }^{2}\end{array}\right]$

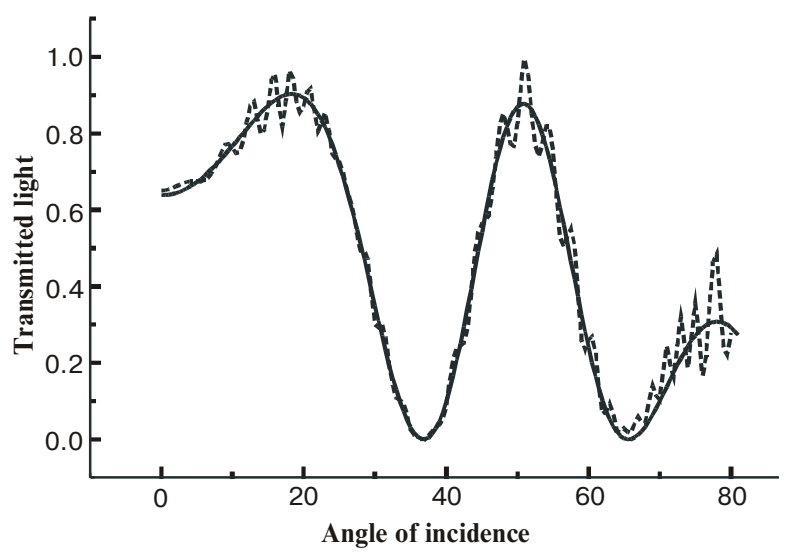

Fig. 2. Transmitted light versus an angle of incident. (Solid curve is the result of Jones' calculation without multi-reflections from boundaries, the dash curve obtained by the Berreman method). 


\section{S. Valyukh et al.: Simulation of obliquely incident light propagation through ...}

\section{TN-LC medium}

The results that were obtained in the previous sections may easily be extended for the finding of the Jones matrix of TN-LC medium and the light intensity transmitted through it. As we have noticed above, TN-LC can be considered as a stack of thin uniaxial plates. The Jones matrix of such system is a product of the Jones matrices of each plate. Although the obtained Jones matrix of anisotropic plate in Eq.(14) is exact, from the viewpoint of practice, we shall use the result of Eq.6, find transmitted light intensity and then take from it the light intensity reflected at the first interface (isotropic medium - uniaxial crystal). There are two reasons for this approach. First, the orientations of the optic axes of two neighboring plates very slightly differ one from another. That is why, the reflections at the interfaces between them are almost absent. Second, calculations of transmissions coefficients need a lot of time. And, in this case, we may loss all advantages in comparison with the Berreman method. In this case the Jones expression for TN-LC cell is:

$$
J=\prod_{k=N}^{1} J_{k}=A^{N} \prod_{k=N}^{1}\left(\begin{array}{cc}
B_{k}\left(1-C_{y_{k}}{ }^{2}\right)+1 & B_{k} C_{y_{k}} \sqrt{1-C_{y_{k}}{ }^{2}} \\
B_{k} C_{y_{k}} \sqrt{1-C_{y_{k}}^{2}} & B_{k} C_{y_{k}}^{2}+1
\end{array}\right)
$$

where the index $k$ relates to a correspond plate, $A$ and $B$ are the same as in Eq.6.

For finding the Jones matrix (20), we must correctly describe the orientation of the optical axis versus distance from one boundary to an other $(\vec{C}(z))$. In the relaxed state with no applied field, the orientation of molecules, as a rule, linearly twists from this distance. When an electrical field of sufficient strength is applied, the molecules away from the surfaces tend to realign in a direction approximately parallel to the applied field and normaly to the surfaces. This realignment causes changes in transmission of polarized light, and are successfully used in electro-optical display devices. According to Eqs (7), (8) we find the output intensity without reflections $\left(I_{\text {out }}\right)$. Next, following the same procedures as in [11, 12] we obtain the transmission coefficients $(\hat{T})$. After that we may find the output Jones vector:

$$
\vec{E}_{\text {out }}^{\prime}=\hat{T} \vec{E}_{\text {out }}
$$

To obtain brightness characteristics of the general TNLC display, the passed electrical field ( $\vec{E}_{\text {out }}^{\prime}$ ) must be multiplied by the Jones matrix of analyzer $\hat{J}_{A}($ Eqs 7,19$)$

As a rule, display devices are interesting from the viewpoint of their viewing angle characteristics that are described by equi-contrast ratio curves. A contrast ratio is determined as a ratio of the intensity in an open state (field off) to the intensity in closed state (field on):

$$
C=\frac{I_{\text {open }}}{I_{\text {close }}}
$$

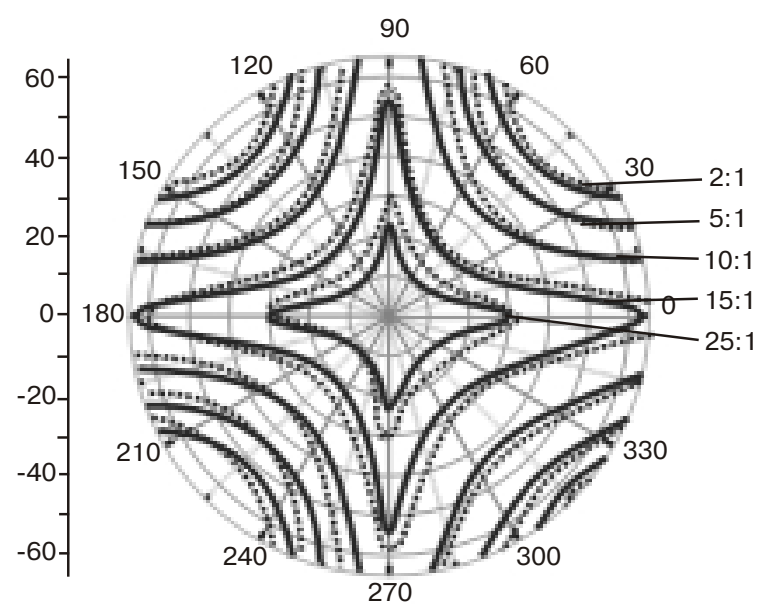

Fig. 3. Equi-contrast ratio curves. Solid curves are the result of calculations, dash curves are experimental data.

Since the orientation of molecules near surfaces depends only on boundary conditions, the Frenel reflection between isotropic medium and LC is not a function of applied external field. That is why, when we determine the contrast ratio, it does not need to define this reflection, because it cancels out. Thus, our problem becomes simpler here. Analysis of the experimental data shows, that the multi-reflections between surfaces of TN-LC are small. So, we may neglect them in our calculations.

In order to examine our calculations, it is reasonable to compare both computed and treated experimental data. We have obtained equi-contrast ratio curves of a $90^{\circ} \mathrm{TN}$ LCD. Here, $5 \mu \mathrm{m}$ cell filled with the Merck 3145 liquid crystal mixture placed between crossed polarisers and driven with a $0 \mathrm{~V}$ - close state and a $15 \mathrm{~V}$ - open state.

Fig. 3 illustrates the derived results. Theoretical calculations are shown by solid curves, experimental data are plotted by dash curves. They were obtained using the Display Measuring System (DMS-408) that has been developed in ISP NASU. Both results are in a good agreement.

Finally, it is noteworthy the computation time of such characteristics, using method proposed by us, is approximately 10 times shorter than that of the fast Berreman method!

\section{Conclusions}

We suggested the way for determining characteristics of light transmitted through a general TN-LCD in the case of oblique incidence using the simple Jones matrix method. The results were successfully tested for uniform anisotropic and TN-LC media. They are in a good agreement with the Berreman propagation matrix technique and experimental data. This method is extremely useful for problems of optimization, when varying parameters in order to improve the viewing characteristics of the conventional $90^{\circ} \mathrm{TN}-\mathrm{LCD}$ and various STN, OMI, as well 


\section{S. Valyukh et al.: Simulation of obliquely incident light propagation through ...}

as LTN-LCDs. In addition, it is also well suitable for the experimental measurement of the cell gap, tilt and twist angles of a general TN-LCD. It can be successfully applied to finding the light propagation through any stratified anisotropic media with small difference between orientations of optical axes in adjacent layers.

This work was supported by STCU under the Contract No. 637 and INTAS under the Grant No. 30234.

\section{References}

1. D. W. Berreman, Opitcs in stratified and anisotropic media 4x4-matrix formulation // J. Opt. Soc. Am. 62, pp. 502-510 (1972).

2. R. M. A. Azzam and N. M. Bashara, Simplified approach to the propagation of polarizated light in anisortropic media application to liquid crystals // J. Opt. Soc. Am. 62, pp. 12521257 (1972).

3. C. H. Gooch and H. A. Tarry, The optical properties of twisted nematic liquid crystal structures with twist angles less then $90^{\circ} / /$ J. Appl. Phys. D, 8, pp. 1575-1584 (1975).
4. H. L. Ong, Origin and characteristics of the optical properties of general twisted nematic liquid-crystal displays // $J$. Appl. Phys. 64, (2), pp. 614-628 (1988).

5. R.C. Jones, Matrix formalism in optical systems // J. Opt. Soc. Am. 31, p. 488 (1941).

6. P. Yeh, Optical properties of general twisted-nematic liuidcrystal displays // J. SID 5/3, pp. 289-292 (1997).

7. S. Valyukh, A. Slobodyanyk, V. Sorokin, Computer simulation of light propagation through twisted NLC cells // Proc. of Vl-th Int. Sympoz., pp. 76-79, Crimea, Ukraine(1997).

8. S. Chanderasekhar, Liquid Crystals, Cambrige University, Cambrige, 1977.

9. H. Wohler, G. Haas, M. Fritsch, D.A. Mlynski, Faster $4 \times 4$ matrix method for uniaxial inhomogeneous media // J. Opt. Soc. Am. 5, pp. 1554-1557 (1988),

10. M. Born and E. Wolf, Principles of optics, Pergamon, New York, 1970

11. F.I. Fedorov, Otrazhenie i prelomlenie sveta prozrachnymi kristallami, Nauka i tekhnika, Minsk, 1975.

12. J. Lekner, Brewster angles in reflection by uniaxial crystals / / J. Opt. Soc. Am. A10, pp. 2059-2064 (1993). 